\title{
Beam divergence studies on hard edge unstable resonators for a long pulse $\mathrm{XeCl}$ excimer laser
}

\author{
R.M. Hofstra. F.A. van Goor, W.J. Witteman \\ Twente Universin, P.O. Box 217. 75f(0) AE Enschede. The Netherlemds
}

Received 10 February 1997: revised I July 1997: accepled 2 July 1997

\begin{abstract}
The focusability of a long pulse $\mathrm{XeCl}$ excimer laser has been improved using confocal positive branch unstable resonators where the outcoupling is done through the convex mirror. A nearly diffraction limited output beam is obtained from hard edge unstable resonators. An improvement of the far field encrgy distribution is achieved with partial reflecting hard edge outcoupling mirrors. The phase delay between the central part of the heam and the beam edge caused hy these partial reflecting mirrors can he reduced by using phase unifying mirrors. Using unstable resonators a hrightness of $1 .+\times 10^{15} \mathrm{~W} / \mathrm{cm}^{2}$ st has been obtained from a long pulse XeCl excimer laser 1997 Elsevier Science B.V.
\end{abstract}

\section{Introduction}

The divergence of the beam from a long pulse $\mathrm{XeCl}$ laser is of the utmost importance for industrial applications. The active medium of this excimer laser has in practice usually large discharge dimensions. Thus it is difficult to have a fundamental Gaussian beam that fills the whole discharge volume. The beam from a stable planoconcave resonator is therefore multimode having a relalively perr divergence. To decrease the divergence the number of oncillating modes must be decreased to, preferahly. one fundamental mode with dimensions that fill as much ar possible the available gain volume.

In the past different unstable resonator configurations have heen successfully applied for shon pulse XeCl lasers $[1-5]$ wrohtain this situation of a low number of oscillating modes. These lasers alre characterised by discharges with high power density and hence short duration ( $\leq 50 \mathrm{~ns}$ ) in order to prevent the onset of discharge instabilities during the latser pulse. This meanls a high gain and only a few car ity round trips for the optical pulse. The optical output from these short pulse lasers is given by the superposition of sutputs from each cavity round trip [6] and has theretore low spatial coherence. The divergence of the optical pulse cill be decreased considerably if an active medium with a lower gain and innger stability period is used instead. so that more round trips are used to reasch saturation. With such a system the mode can be established belere salurattion of the gain medium occurs, resulting in a beam with a lower divergence. Only a few investigations have heen carried out in this discharge regine [7-9]. In this article we present a more detailed study of the bean diverence of a $\mathrm{XeCl}$ excimer laser operated in the long pulse. low gain discharge regime.

For our system, having a relatively low gain (3-4t\% $\mathrm{cm}^{-1}$ ) and a long gain lifetime (approximately $250 \mathrm{~ms}$ ). is positive branch confocal unstable resomator in found to he an excellent choice. The negative hranch unstable resonators have an intracavity fecus which might lead (1) problems with optical breakdown. The sutcoupling from a positive hranch unstable resomator is donte at the comsex mirror to get a cullimatted sutput beam. The present article deals with experimental studies of various hard edge resonator comfigurations for a low gain. leng aptical pulse system.

\section{Experimental configuration}

The experiments have been performed with a XeCl laser system having a $2.5 \mathrm{~cm} \times 2 \mathrm{~cm} \times 60 \mathrm{~cm}$ (electrode distance $x$ width $\times$ length) $X$-ray preionised high pres- 


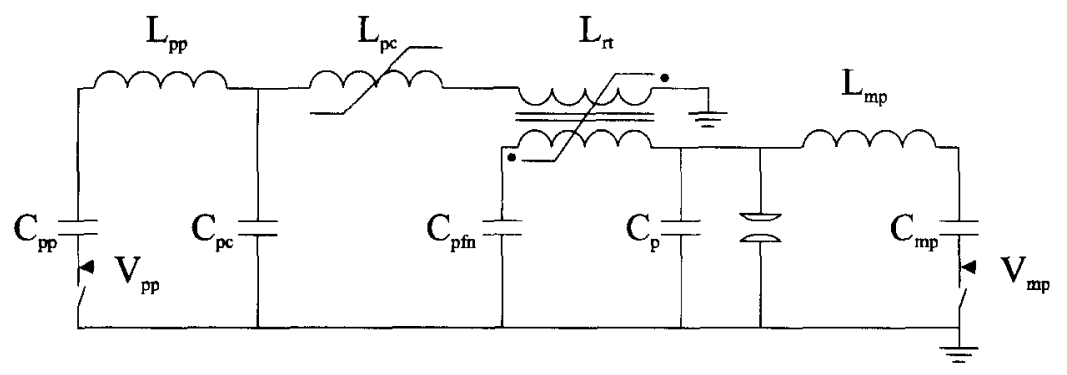

Fig. I. Electrical circuit of the XeCl laser. Typical capacitor values: $C_{\mathrm{mp}}=370 \mathrm{nF}, C_{\mathrm{ptn}}=388 \mathrm{nF} . C_{\mathrm{rr}}-5.1 \mathrm{nF} . C_{\mathrm{pc}}=3.4 \mathrm{nF}$ and $C_{\mathrm{F}}=3.6$ $\mathrm{nF}$.

sure discharge [10]. The gas mixture used during the experiments consisted of approximately $1 \mathrm{hPa} \mathrm{HCl}, 10 \mathrm{hPa}$ $\mathrm{Xe}$ and $\mathrm{Ne}$ as buffer gas up to a pressure of $0.5 \mathrm{MPa}$. The discharge was excited by a spiker-sustainer circuit using a 'race-track' saturable inductor as the low-inductance main switch. The 'race-track' is also used as a pulse transformer for the spiker. Fig. 1 shows the electrical circuit of our laser. The laser operates in the resonant overshoot mode [11]. Typical waveforms of the X-ray preionisation pulse. discharge voltage and discharge current are shown in Fig. 2. The efficiency of the laser is maximal if the system operates under matched conditions, i.e. if the voltage on the pulse forming network (PFN) equals twice the steady state voltage of the discharge. The output energy can be increased quite easily by increasing the PFN voltage but then the efficiency drops.

Fitted with a stable resonator consisting of a concave HR rear mirror (radius of curvature $10 \mathrm{~m}$ ) and a flat outcoupling mirror with a reflectivity of $50 \%$ the output energy is approximately $450 \mathrm{~mJ}$ under matched discharge conditions. Experiments with different outcoupler reflectivities have shown that a reflectivity near $20 \%$ is needed to get a resonable output with a stable resonator. Below this minimum value the output drops dramatically.

For the near field measurements the attenuated output beam is imaged on a scintillator using a relay imaging

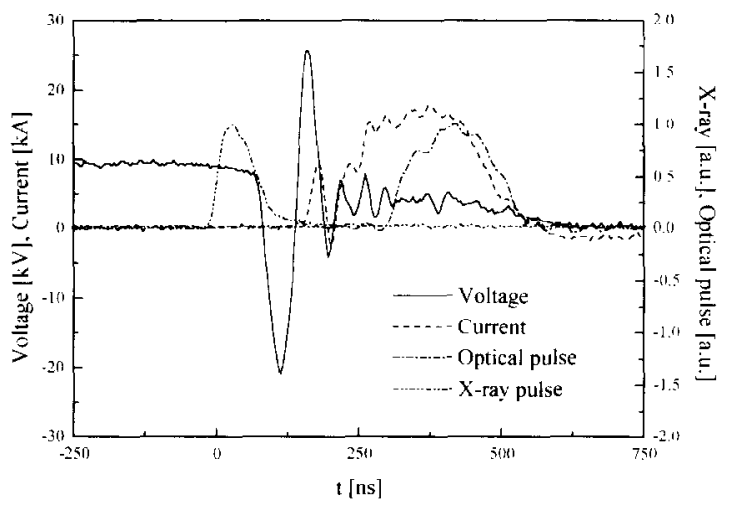

Fig. 2. Typical waveforms of the discharge voltage, discharge current, X-ray preionisation pulse and optical output pulse. system of two lenses with a focal length of $50 \mathrm{~cm}$ placed 1 $\mathrm{m}$ apart. The scintillator used is a thin film of a solution of sodium salicylate in water between two quartz windows. The scintillator shows a linear response to the UV input signal as long as the intensity is kept low. In our near field measurements only about $1 \%$ of the beam power is allowed to fall on the scintillator to prevent nonlinear effects due to high power densities in the focus of the relay imaging system. In this intensity region the scintillator response is found to be linear with the laser intensity. However, the image intensified CCD camera which was used to obtain the image is found to have a somewhat nonlinear response. Experiments showed that the camera response is slightly nonlinear with the incident intensity. Lower intensities have a relatively larger response than higher intensities. This nonlinearity is found to be related to the CCD and not to the image intensifier so that the obtained image can be corrected for this nonlinearity using a correction function.

The far field pattern is measured by focussing the attenuated beam (attenuation $\leq 0.05 \%$ ) on the scintillator using a concave mirror with $10 \mathrm{~m}$ radius. To obtain the image the CCD camera has been equipped with a microscope objective.

Due to the small gate time of the camera, down to $5 \mathrm{~ns}$, it is possible to look at the time evolution of the optical pulse which lasts approximately $200-250 \mathrm{~ns}$ when a stable resonator is used and approximately $150 \mathrm{~ns}$ when unstable resonators are used. Fig. 2 shows a typical waveform for the optical output pulse when a stable resonator is used.

\section{High reflectivity hard edge unstable resonators}

Hard edge unstable resonators are resonators where the outcoupling mirror shows a step in the reflectivity profile. If the central spot on the outcoupling mirror has high reflectivity (HR) the outcoupled near field is ring-shaped with practically no energy in the center of the beam. Fig. 3 shows the near field beam profile for a resonator with a geometric magnification $M$ of 2.4 and a central HR spot 

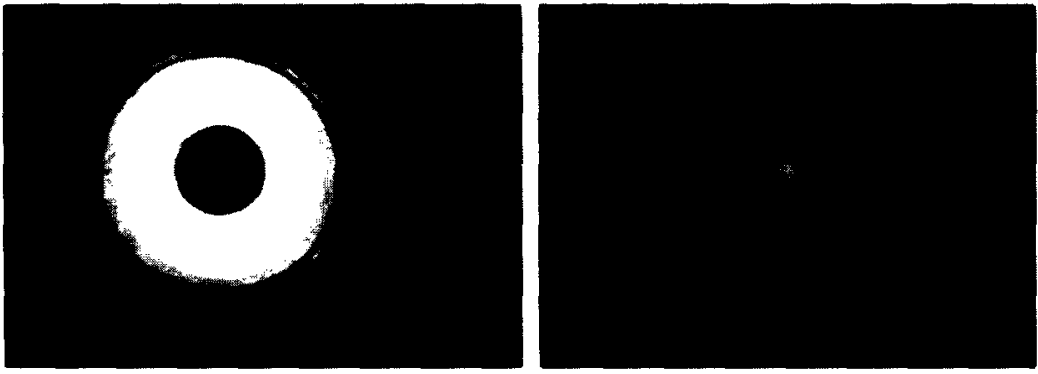

Fig. 3. Near field beam profile (left) and focus profile (right) of the beam from a hard edge unstable resonator $(M=2.4)$ with a central HR spor of $8 \mathrm{~mm}$ diameter focussed by a $10 \mathrm{~m}$ radius concave mirror.

of $8 \mathrm{~mm}$ diameter. The diffraction pattern at the beam edge is caused by the hard mirror edge which may not be perfect due to the fabrication process of the HR coating.

The choice for a magnification $M=2.4$ is based on the minimum feedback criterium found in the stable resonator measurements mentioned above. There we found that at least about $20 \%$ feedback is needed to get a proper energy extraction. The value of 2.4 for the magnification results in a geometrical feedback of $17 \%\left(\gamma=1 / M^{2}\right)$ which is just below this value.

An approximation of the eigenmodes of a hard edge resonator can be determined using the virtual source theory [12]. Calculation of the lowest order mode of our resonator results in the near field energy distribution just behind the outcoupling mirror shown in Fig. 4. A measured near field energy distribution is also shown in this figure. The calculation is based on an unloaded resonator. i.e. the gain medium is not taken into account. The gain medium smoothes the profile due to the saturation of the gain [12].

If a gated camera is used to look at the time-behaviour of the near field energy distribution it is found that the near field energy profile does not vary strongly during the pulse. The profile has build up before saturation of the

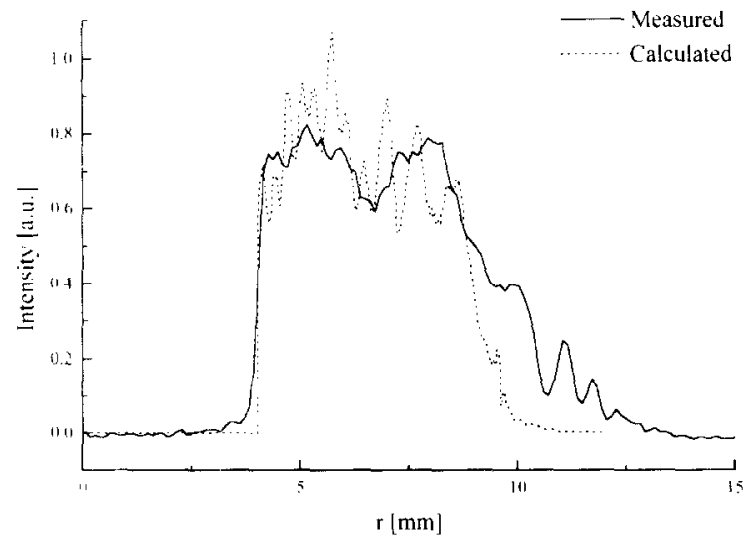

Fig. 4. Calculated and measured near field energy distributions of a hard edge unstable resonator with $M=2.4$ and a central HR spot ol $8 \mathrm{~mm}$. gain and remains unchanged during the output pulse. The small variations that could be seen were found to be small shot-to-shot variations. The focus energy distribution shows more variations but this occurs because the lowest order mode does not have build up completely at the beginning of the optical beam. Thus small phase and amplitude variations can occur in the near field. Especially these phase variations have their effect in the far field. But the focus measurements show. on average, no large differences between the beginning of the pulse and the end of the pulse.

The measured far field energy distribution of the beam from the HR unstable resonator is shown in Fig. 3. The far field energy distribution shows ring structure as expected from the diffraction of a cylindrical beam. A cross-section of the focus is shown in Fig. 5. From the above calculated outcoupled field energy distribution the far field energy distribution can be calculated by propagating the beam through a lens to the focal plane of this lens. This propagation of the wavefront is given by the Huygens integral [12]. In cylindrical coordinates the Huygens integral can be evaluated using the Fast Hankel Transform method [1.3] if the wavefront $u(r . \phi)$ is separable with respect to the variables $r$ and $\phi$. The result of this calculation is also shown in Fig. 5. It is seen that the measured focus energy

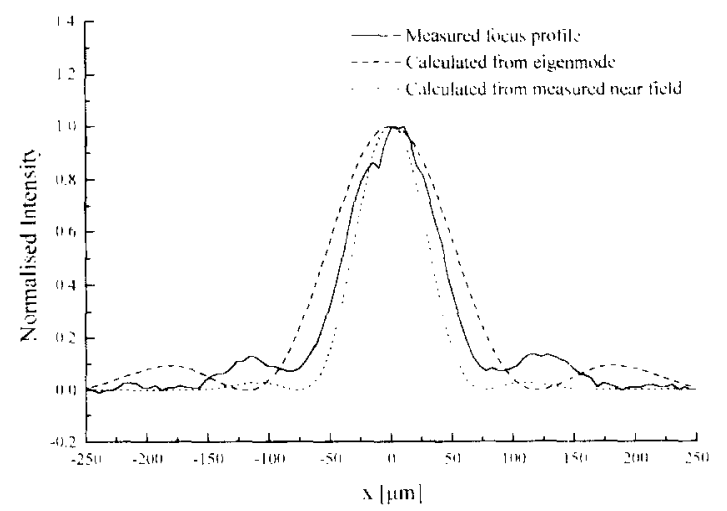

Fig. 5. Comparison between far field energy distributions from theory and experiments. 
distribution is slightly narrower than the calculated focus energy distribution. This is due to the smoother and somewhat wider near field energy distribution. However, if we calculate the focus energy distribution from the measured near field energy distribution shown in Fig. 4 using a uniform phase distribution a smaller focus is found as can be seen in Fig. 5. The measured focus energy distribution is slightly wider than the calculated distribution thus the output beam is nearly diffraction limited. The FWHM of the measured focus is $16.7 \mu \mathrm{rad}$ and the FWHM of the calculated focus is $13.1 \mu \mathrm{rad}$.

The TDL-parameter (Times Diffraction Limited) introduced by Bollanti et al. [14] is in our view a proper parameter to describe the beam quality as it is based on real beams instead of ideal Gaussian beams. The TDLparameter is defined as

$\mathrm{TDL}=\frac{\theta_{\text {meas }}}{\theta_{\text {caltc }}}\left(\frac{k_{\text {calc }}}{k_{\text {meas }}}\right)^{1 / 2}$,

where $\theta_{\text {meas }}$ and $\theta_{\text {valc }}$ are the measured and calculated beam divergence respectively, and $k_{\text {meis }}$ and $k_{\text {calc }}$ the respective energy content within that divergence angle.

The full divergence angle based on the first minimum is $32 \mu \mathrm{rad}$ for the measured beam and $30 \mu \mathrm{rad}$ for the calculated heam. The measured heam has approximately $60 \%$ of the energy within the central peak and the calculated beam $82 \%$ of the energy. This results in a TDLparameter of 1.3 for our beam.

The output energy is $339 \mathrm{~mJ}$ in a $1+6 \mathrm{~ns}$ pulse under matched discharge conditions. Approximately $60 \%$ of this energy is focussed in the central peak. This results in a brightness of $5.5 \times 10^{14} \mathrm{~W} / \mathrm{cm}^{2} \mathrm{sr}$. If the discharge is pushed to its limits the pulse energy can be increased to approximately $850 \mathrm{~mJ}$, leading to a brightness of $1.2 \times 10^{15}$ $\mathrm{W} / \mathrm{cm}^{2} \mathrm{sr}$. In both cases a large part of the energy, about $40 \%$. is lost in the side lobes of the focus intensity profile. This results in a brightness that is lower than the brightness that could be obtained if all encrgy would be in the central peak.

\section{Partial reflecting hard edge unstable resonators}

Calculations predict that the side lobe energy can be reduced by lowering the reflectivity of the central spot on

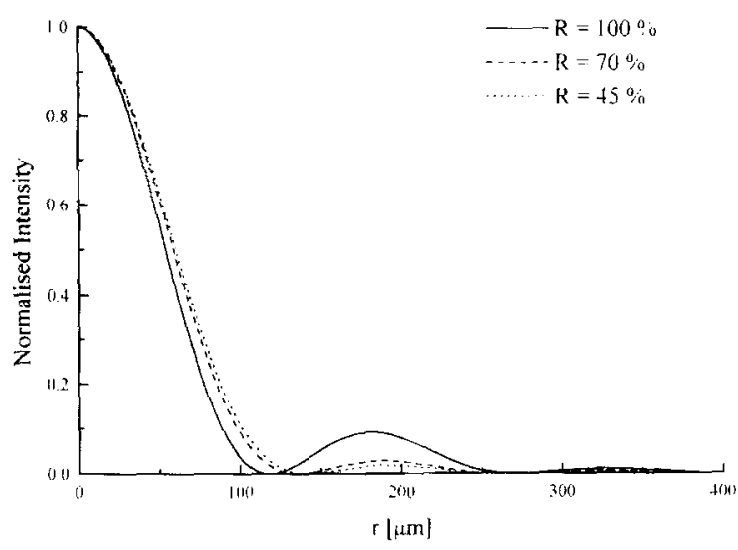

Fig. 6. Calculated focus energy distributions with different central reflectivities for the outcoupling mirror.

the outcoupling mirror. Fig. 6 shows the calculated focus energy distributions based on the lowest order eigenmode of a hard edge resonator with a magnification $M$ of 2.4 and an outcoupling mirror radius of $4 \mathrm{~mm}$. It can be seen that if the central reflectivity is lowered the side lobe is also reduced. The width of the central peak however increases slightly, thus the beam of a partial reflecting hard edge unstable resonator will show a slightly larger divergence than a high reflectivity hard edge resonator.

Table I shows the characteristics of different resonators used in the experiments. $R$ in Table 1 is the reflectivity of the reflecting area and $D_{\mathrm{r}}$ its diameter. The geometrical feedback $y=R / M^{2}$. which gives only an indication of the actual feedback, is determined by the magnification $M$ and the reflectivity of the central area $R$ and is independent of the size of the reflecting area. $\theta_{i}$ is the full divergence angle based on the first minimum in the focus energy distribution. $E$ the output energy under matched discharge conditions. $\tau$ the pulse duration. $k$ the percentage of energy within the central peak and $B$ the corresponding brightness, which is defined as

$$
B=k \frac{E}{\tau}\left(\pi \frac{\theta_{t}}{2} \frac{M D_{\mathrm{r}}}{2}\right)^{-2}
$$

Fig. 7 shows the distribution of the energy in the focus for the three resonators with the same magnification $M=$

Table 1

Characteristics of and experimental results obtained with the laser equipped with different unstable resonator configurations and operated under matched discharge conditions

\begin{tabular}{|c|c|c|c|c|c|c|c|c|}
\hline$M$ & $R[\%]$ & $D_{r}[\mathrm{~mm}]$ & $\gamma[\%]$ & $\theta_{d l}[\mu \mathrm{rad}]$ & $E[\mathrm{~mJ}]$ & $\tau[\mathrm{ns}]$ & $k[\%]$ & $B\left[\mathrm{~W} / \mathrm{cm}^{2} \mathrm{sr}\right]$ \\
\hline 2.4 & 100 & 8 & 17.7 & 32 & 339 & 160 & $\overline{60}$ & $5.5 \times 10^{14}$ \\
\hline 2.4 & 72 & $x$ & 12.5 & 37 & 334 & 146 & 70 & $4.6 \times 10^{14}$ \\
\hline 2.4 & 45 & 8 & 7.8 & 31 & 305 & $1+6$ & 50 & $4.1 \times 10^{1+}$ \\
\hline 2.0 & 72 & 10 & 17.5 & 36 & 362 & 149 & 70 & $5.0 \times 10^{14}$ \\
\hline 1.6 & 45 & 12 & 17.5 & 34 & 359 & 160 & $30)$ & $2.5 \times 10^{14}$ \\
\hline
\end{tabular}




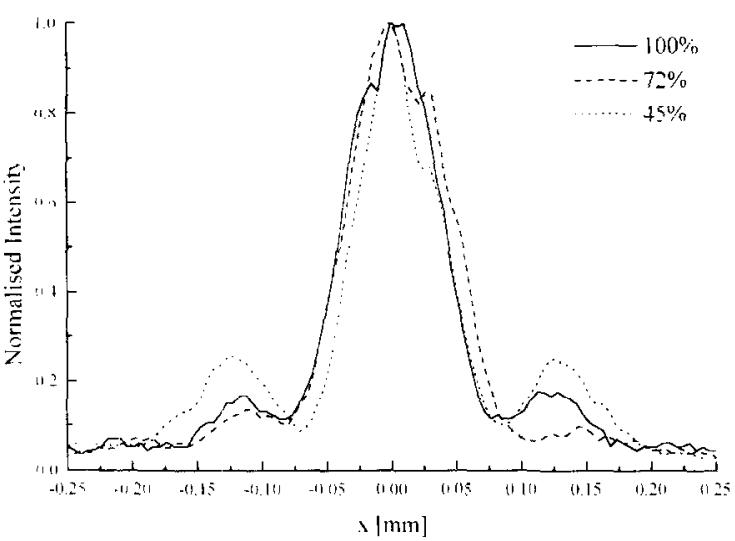

Fig. 7. Focus energy distributions from resonators with different central retlectivities $(100) .72$ and $45 \%)$. Magnification $M=2.4$ for all resomators.

2.4. mentioned in Table 1. These resonators are identical (same radii of curvature for both mirrors and same resonator length) except for the reflectivity of the central spot ( $1(0), 72$ and $45 \%)$. It is seen that the $72 \%$ spot reflectivity shows an improvement of the energy distribution in the focus: the energy in the side lobe is lowered (approximately $70 \%$ of the pulse energy is found in the central peik). However the width of the focus is slightly larger and the pulse energy slightly lower due to the lower feedback so that the brightness decreases slightly. At $45 \%$. retlectivity the side lobe energy increases to a level above the $100 \%$ reflection case. This is probably caused by the outcoupler: due to the coating used to obtain the reflectivity profile there will exist a phase difference between the central transmitted part and the edge of the beam. This phase difference results in more energy in the side lobe. Interferometric measurements show that the $70 \%$ mirror has a phase difference of approximately $0.3 \pi$ and the $45 \%$ mirror a phase difference of approximately $\pi$. These values are in agreement with those expected from the coatings on the mirtors. The phase difference introduced by the couting can be reduced hy using phase unifying mirrors [15.16]. This will be discussed in Section 5.

When the reflectivity of the central part is lowered the geometrical feedback $\gamma$ is also lowered. To keep the gerometricil feedback similar for different reflectance val ues the resonator magnification has to be decreased for decreasing mirror reflectance. So experiments have been performed using resonators with equal geometrical feedback. To ensure a proper filling of the gain volume the size of the reflecting spot on the outcoupling mirror was also increased. This however has a negative effect on the diveruence. The focus from the resonator with a larger mirror size is somewhat larger so that the brightness will be reduced somewhat. However, if we would have chosen to keep the minor size the same the brightness would also be reduced due to the smaller outcoupled beam $(M D$, is reduced) and the energy extraction would be reduced because of the worse gain volume usige.

The characteristics of these resonators can be found in Table 1. The obtained results are similar to the results with the resonators having the same magnification mentioned above. A smooth. but slightly wider focus profile (as expected. see above) with practically mo side lobes is obtained for the resonator with an outcoupler of $72 \%$ reflectivity and the resonator fitted with an outcoupler having a central reflectivity of $45 \%$ leads to more side lobe energy than the resonator with $100 \%$ reflecting area. The focus from this last resonator is shown in Fig. 8. The figure shows a clear asymmetry between the horizontal and vertical directions. This effect is most distinct for this specific resonator and the phase unifying resonator of the next paragraph. The focus images from the other resonators treated before showed only a very small asymmetry and look similar to the one depicted in Fig. 3. This small asymmetry in the focus energy distribution probably originates from the small asymmetry which can be found in the near field entrgy distribution. The latter might be caused by a nonhomogeneous discharge due to a nonuniform deposition of the electrical power into the discharge.

However. Fig. 8 shows a rather large asymmetry. which is not present in the near field energy distribution. We think that this asymmetry might be caused by the elecrodes. During the start-up of the system several modes try to uscillate. Due to different losses the respective modes have strong competition. In general the inode with the lowest losses will reach the highest intensity and will suppress the weaker modes. For survival of this strongest mode with the lowest losses several uncillations between the mirrors have to take place before saturation of the gain medium. In the case of a low magnification system there are relatively lower losses for the higher order modes so that the competition process becomes slower and it takes more oscillations for the lowest loss mode to dominate. If the gain medium saturates before the end of the mode competition. as in pulsed systems like ours, the output beam still contains higher order modes. This means that the far field energy distribution has a larger spot size. Iu the case of the $M=1.6$ resonator the gain medium is probably saturating too early so that not only the lowest loss mode but also a few other modes are present in the beam. The losses for these higher order modes are different for the horizontal and the vertical direction. In the horizontal direction the gain volume is restricted by two hard edges: the electrodes. In the vertical direction there is no hard restriction as the gain volume ends where the discharge ends: at the border of the preionisation, which is a rather soft transition. Thus in the horizontal direction the losses for the higher order modes are larger than in the vertical direction. resulting in a beam which shows less higher order modes in the horizontal direction than in the vertical direction. This results in all asvmmetrical focus 
having more side lobes in the vertical direction than in the horizontal direction.

\section{Phase unifying unstable resonators}

If a beam passes a partial reflecting hard edge outcoupling mirror a phase difference between the central part (i.e. the part that passes through the reflecting coating) and the outer part (i.e. the part that passes beside the reflecting coating) can occur. This transmission phase difference between the central spot and the rest of the mirror can be decreased by using the so-called phase unifying mirrors $[15,16]$. As mentioned before the $45 \%$ outcoupling mirror showed a phase difference of nearly $\pi$. Thus experiments were performed with a resonator with a magnification of $M=1.6$ having a phase unifying outcoupling mirror with a central reflectance of $45 \%$. Interferometric measurements on this mirror show only a very small phase difference. Calculated from the coating there is a phase difference of only $0.16 \pi$. Fig. 8 shows typical results. It is clearly seen that the output beam from the phase unifying resonator has a better focus than the non-phase unifying resonator. The non-phase unifying resonator shows a lot more side structure than the phase unifying resonator. The output energy from the phase unifying resonator is a little higher than from the normal hard edge resonators: $399 \mathrm{~mJ}$ in a $160 \mathrm{~ns}$ pulse. The diffraction angle of the beam is comparable to the diffraction angle as mentioned in Table 1 for the $m=1.6$ resonator with a $45 \%$ outcoupler: $34 \mu \mathrm{rad}$, however approximately $80 \%$ of the energy is found in the central peak instead of less than $30 \%$ for the standard mirror resonator. Thus resulting in higher brightness: 7.0 $\times 10^{1+4} \mathrm{~W} / \mathrm{cm}^{2}$ sr under matched discharge conditions and $1.4 \times 10^{15} \mathrm{~W} / \mathrm{cm}^{2} \mathrm{sr}$ at maximum energy loading of the discharge.

\section{Conclusions}

It has been demonstrated that with a high reflectivity hard edge unstable resonator having a magnification of 2.4 a nearly diffaction limited beam (TDL $=1.3$ ) can be obtained from our low gain, long pulse XeCl-excimer laser. Under malched discharge conditions the brightness of the pulse is $5.5 \times 10^{1+} \mathrm{W} / \mathrm{cm}^{2} \mathrm{sr}$. The maximum obtained brightness was $1.2 \times 10^{15} \mathrm{~W} / \mathrm{cm}^{2} \mathrm{sr}$.

The side lobe energy in the far field energy distribution can be reduced by using partial reflecting outcoupling mirrors. This leads to a small increase in divergence angle. but if the geometrical feedback is kept constant the brightness of the beam increases due to higher central peak energy. However, in the case of partial reflecting outcoupling mirrors the coating can cause phase problems as we have seen with the $45 \%$ mirrors. It has been shown that these phase problems can be avoided by using phase unifying mirrors. Due to lower side lobe energy and lower diffraction effects at the beam edge the brightness of the beam from a $45 \%$ reflectivity phase unifying resonator with a magnification of 1.6 is $7.0 \times 10^{1+} \mathrm{W} / \mathrm{cm}^{2}$ sr. The maximum obtained brightness with the phase unifying resonator was $1.4 \times 10^{15} \mathrm{~W} / \mathrm{cm}^{2} \mathrm{~s}$.

\section{Acknowledgements}

The present work has been supported by the Netherlands Technology Foundation (STW).

\section{References}

[1] V. Boffa. P. di Lazzaro, G.P. Gallerano, G. Giordano, T. Hermsen. T. Letardi, C.E. Zheng. IEEE J. Quantum Electron. 23 (1987) 1241.

[2] A. Luches, V. Nassisi, M.R. Perrone. Appl. Optics 28 (1989) 2047.

[3] M.R. Perrone, F. Mezzolla, C. Cali, C. Pace, Appl. Physics Lett. 59 (1991) 1153.

[4] M.R. Perrone, A. Piegari, S. Scaglione, IEEE J. Quantum Electron. 29 (1993) 1423.

[5] M.R. Perrone. Pure Appl. Optics 3 (1994) 523.

[6] M.R. Perrone, C. Palma. V. Bagini, A. Piegari. D. Flori, S. Scaglione, J. Opt. Soc. Am. A 12 (1995) 991.
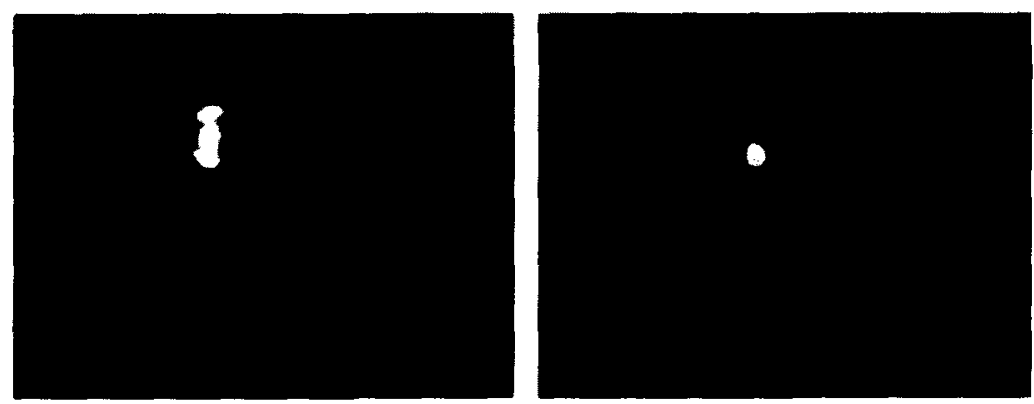

Fig. 8. Focus spots from a hard edge resonator with magnification of 1.6 and a central spot reflectance of $45 \%$ fitted with a standard mirror (left) and fitted with a phase unifying mirror (right). 
[7] T.J. McKee, Appl. Optics 301991635.

[8] T.J. McKee, S. Fendrykowski. Appl. Optics 32 (1993) 275.

[9] S.E. Kovalenko. V. Lonev. M.R. Perrone. Appl. Optics 33 (1944) 4082.

[10] J.C.M. Timmermans. Ph.D. Thesis. University of Twente. Enschede, The Netherlands, 1995.

[11] I.W Gerritsen, A.L. Keet. G.J. Ernst, W.J. Witteman, J. Appl. Physics 67 (1990) 3517.
[12] A.E. Siegman, Lasers (University Science Boohs. Mill Valley. CA 94941, USA. 1986).

[13] A.E. Siegman. Optics Lett. 1 (1477) 13.

[14] S. Bollanti. P. di Lazarro. D. Murra, Optics Comm. 134 (1997) 503.

[15] V.B. Kaul. S.V. Mel'chenko, M.R. Perrotke, A. Piegari, V.F. Tarasenko. J. Mod. Optics $42(1945) 2279$.

[16] M.R. Perrone. Optics Comm. 116 (1995) 101 